significant relationships with hydration status, symptoms and survival in advanced cancer. However, further work is needed to study these associations in the dying.

Aim This feasibility study aimed to develop the necessary methodology to evaluate hydration and its relationship with clinical symptoms and quality-of-life in dying cancer patients.

Methodology An observational study of thirty patients with advanced cancer in a hospice and hospital-based specialist palliative care inpatient unit. An advanced consent methodology was used to conduct assessments in the dying phase. Assessments involved hydration (BIVA), symptoms, physical signs, quality of life, myoclonus and survival. Family-caregivers experiences of hydration were evaluated via questionnaire.

Results The feasibility aspect of the study was successful in recruiting patients and caregivers across the research sites. The overall recruitment rate for patients was 60\% (30 out of 48 approached agreeing to participate) with six (20\%) of those recruited receiving a further hydration assessment in the dying phase of their illness. Eighteen caregivers completed questionnaires.

Conclusion It is feasible to use BIVA to assess hydration in the dying. This study will support the next phase of the study which include recruitment from additional palliative care units. The outcomes from this work will help to identify hydrationassociated variables to support development of a clinical hydration assessment tool. Ultimately, this will help to develop a framework to clinically assess and manage hydration states patients with cancer.

\section{PRINCIPLES OF CARE FOR THE DYING PATIENT- AUDIT OF USE IN A TERTIARY REFERRAL CANCER CENTRE}

A Halley, J Ramsay, H Johns, G Kane, A Stephens, AM Stevens. The Royal Marsden NHS Foundation Trust

\subsection{6/bmjspcare-2019-ASP.58}

Background The Leadership Alliance for the Care of Dying People set out an approach with focus on achieving the five priorities of care. Our trust developed an electronic version of the London Cancer Alliance document, The Principles of Care for Dying Patients. This audit set out to determine the number of expected deaths who had a document in place and if completed, adherence to the NICE Quality Standards for care of adults in the last days of life (NG31).

Methods Prior to the National Care at the end of life Audit, the hospital palliative care team undertook an audit of care of patients in the last days of life for deaths from 1st April 2017 to 31st March 2018. There were 230 deaths across the trust. Deaths in critical care and paediatric deaths were excluded. 177 case record reviews were undertaken by the palliative care team.

Results $65 \%$ of patients who were recognised as dying had a principles of care document initiated.

NICE Quality Standard 1: 100\% of patients had daily review to assess for changes in dying phase, i.e. nearing death, stabilising or recovering.

NICE Quality Standard 2: 53\% of patients had capacity to be able to discuss, and review an individualised care plan (ICP). 97\% of cases showed documented evidence of a family discussion to ensure communication of recognition of dying and development of ICP.

NICE Quality Standard 3: - 94\% of patients had appropriate anticipatory medications prescribed.
NICE Quality Standard 4\%-68\% of patients had documented evidence of discussion of hydration status.

Conclusion The Principles of Care Document when used adheres to the Nice Quality Standard. The regular education of the multi-disciplinary team in caring for dying patients continues to focus on use of the document to direct excellent care of the dying.

\section{DESCRIBING DEPRESCRIBING - WHEN ARE WE STOPPING MEDICATIONS IN PALLIATIVE CARE?}

Matthew Doré, Trish Campbell, Derek Willis. Severn Hospice

\subsection{6/bmjspcare-2019-ASP.59}

Introduction Deprescribing is the process of withdrawal of medication with the goal of improving patient care. Research currently is within the context of polypharmacy and geriatrics, however despite its ubiquity within palliative care it has not been studied significantly. Anecdotally, deprescribing is performed adhoc and using 'common sense' but there appears no clear data on rational and benefit.

Aim To gather data on current deprescribing practice within palliative care, with the aim to inform our own specialty and others.

Methodology Prospectively palliative in-patient hospice and community patients had medications which were deprescribed recorded and the rational. This gained a timeline of medication stops respective to date of discharge and/or death.

Results Data collected from 13/3/18 to 30/06/18 with 647 medication stops of 197 differing medications. 56\%(361) medication stops were due to approaching end-of-life. 15\%(93) due to swallowing difficulties, 17\%(109) due to 'rationalising' medications.

The median number of days of palliative care deprescribing any medication before death is 4 , with 1 st quartile $(25 \%)$ being 1 day and quartile 3 (75\%) being 9 days before death. Groups identified and measured, included statins (median 5 days), beta-blockers (median 2 days), ACEi (median 5 days), anti-platelets (median 9 days), and Warfarin/NOACS (median 5 days).

Conclusion/discussion By describing deprescribing we outline the short timespan between medication stopping and death for arguably minimal patient benefit at this point of life. The rational for stopping correlates with this postulation, outlining we are stopping the vast majority of medications due to the dying phase rather than preemptively. Arguably, gathering data alone might inform practice but does not in itself bring about change. We suggest therefore that this study should therefore be seen as a positive step into identifying the need for change in current practice, leading to future studies to outline how change is achieved and measured.

\section{THE USE OF PERSONALISED END OF LIFE CARE PATHWAY AT THE ROYAL SURREY COUNTY HOSPITAL}

Helen Thomson, Katherine Webber. Royal Surrey County Hospital

\subsection{6/bmjspcare-2019-ASP.60}

Introduction National guidance has recommended locally developed personalised care plans to ensure needs are met as patients are approaching end of life. The Royal Surrey County 
Hospital (RSCH) has developed a Personalised End of Life Care Plan (PELICan). This aim of this project is to assess the use of PELICans in a District General Hospital and Cancer Centre over a three year period.

Methods A database was created of all patients with a PELICan at the RSCH over a three year time period (1/9/2014$31 / 8 / 2017)$. Outcome measures included start date of PELICan, presence of PELICan over a weekend and length of time on PELICan before death or discontinuation.

Results 1378 patients had a PELICan over three years. There was an increasing number of patients placed with a PELICan every year $(53 \%, 55 \%$ and $62 \%$ in successive years). The mean time a patient was cared for with a PELICan before death or discontinuation was 4.5 days (range 1-169 days). Only $7 \%$ of PELICans were discontinued over three years, with $71.4 \%$ being stopped in the first seven days. $16.8 \%$ percent of PELiCans were commenced over the weekend, with this number increasing every year $(14.5 \%, 16.0 \%$ and $19.6 \%$ in successive years). Of those patients who died with a PELICan, $27.2 \%$ died on a weekend.

Conclusion The use of the PELICan is increasing at the $\mathrm{RSCH}$. There has been the introduction of a seven day Palliative care service at the $\mathrm{RSCH}$, which is imperative when considering the proportion of patients dying with a PELICan, as well as those dying on a weekend. Physicians are quite accurate with their prediction of death within the next seven days. The PELICan booklet at the $\mathrm{RSCH}$ requires a daily multidisciplinary review, and consequently, most of the PELICans that are discontinued are done so early on.

\section{QUALITY OF DISCHARGE SUMMARIES FOR PATIENTS SENT HOME FROM HOSPITAL FOR END OF LIFE CARE - A REPEAT AUDIT}

Anirudh Kumar, Sophie Hunter, Ruth England. University Hospitals of Derby and Burton NHS Trust

\subsection{6/bmjspcare-2019-ASP.61}

Background $30 \%$ of patients leaving acute hospitals die within 12 months. When patients leave hospital, the discharge summary letter must give adequate, appropriate information to primary care providers so they can optimise ongoing care. The standard of discharge summaries is variable; evidence suggests that quality improvement projects can enhance their value.

Methods This single centre, retrospective, repeat audit carried out by F2 doctors across an acute hospital examined the quality of 58 discharge letters written between 1 st August and 31st October 2017 for patients discharged home with 'fast-track' support, anticipated to be at the end of their life. Written information in discharge summaries was audited against standards from the Royal College of Physicians as well as supplementary local standards developed for use when patients are believed to be in the last weeks of their life.

Results There is still room to improve the quality of discharge summaries for patients being 'fast-tracked' home. Compared with a baseline audit in 2015, more patients were prescribed anticipatory medications on discharge and more 'sensitive discussions' were documented. However, fewer summaries contained documentation of a patient's preference for place of care and fewer had instructions to GPs regarding care at the end of life. There was no documentation about provision of patient information leaflets.

Conclusions Engaging junior doctors in a repeat audit cycle has an impact on quality of discharge summaries. Stickers to prompt content of discharge summaries have been developed and refined. Additional teaching has been provided to Foundation Year doctors. An information leaflet for patients explaining the fast-track process has been created. A further re-audit is planned in 2019 to determine the impact of these interventions.

\section{ARE WE MAKING THINGS ANY BETTER? ASSESSING THE QUALITY OF A PERSONALISED CARE PLAN FOR THE LAST DAYS OF LIFE WITHIN AN ACUTE HOSPITAL TRUST}

J Winter, B Francis, H McDonald, F Malik. East Sussex Healthcare NHS Trust

\subsection{6/bmjspcare-2019-ASP.62}

Background Following CQC inspection, need for improvement in recognising dying/individualised care planning in one acute NHS trust was highlighted. In response, the Last Days of Life Personalised Care Plan (LDoLPCP) was developed based on NICE guidance and Five Priorities for care of dying person. Implementation audited via multiple methods.

Methods

- Retrospective cross-sectional monthly spot audits Nov 17-Apr 18 in two acute hospitals. Those on LDOLCP identified from SPCT current case-list, ward visits \& mortality database/ bereavement office data. 10 care plans reviewed monthly each site. Two clinicians assessed quality of documentation using a standardised audit tool. Areas covered include: recognition, senior decision maker, anticipatory prescribing etc. Each section subjectively rated (High standard, acceptable, needs improvement, poor). Data collated anonymously. Results analysed using summary statistics. Target level for standards set at $80 \%$ of plans completed to a high/acceptable level.

- Online survey questionnaire emailed to all Trust frontline staff to ascertain views/feedback on LDOLPCP. Qualitative feedback gained from frontline Nursing staff on 20 wards cross-site.

\section{Results}

- 110 care plans reviewed. Mean age 82 years, 56\% female. In $73 \%(80 / 110)$, section on recognition of dying completed to high or acceptable level. Highest scores: $83 \%$ for documentation of senior doctor \& 92.9\% for anticipatory prescribing for pain $\&$ nausea. However in other areas, results fell below target including Emotional/spiritual/cultural assessment $36.7 \%$; daily symptom assessment $43.0 \%$ and Hydration status assessed, $48.5 \%$. Reasons for poor quality documentation include uncertainty over whom best to complete, time pressures, LDOLPCP viewed as one-off document rather than an evolving plan.

- Online survey in progress \& reaudit - results to follow.

Conclusion Audit shows areas of mixed quality documentation. Through staff engagement, targeted improvement areas include better MDT communication and shared responsibility, earlier in-patient emotional/spiritual assessment, developing model LDOLCP examples as resources/benchmark the results are expected to improve. 